ISSN 2075-9827 e-ISSN 2313-0210

Carpathian Math. Publ. 2018, 10 (2), 376-386

doi:10.15330/cmp.10.2.376-386

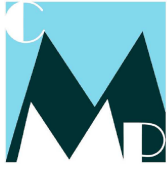

http://www.journals.pu.if.ua/index.php/cmp

Карпатські матем. публ. 2018, Т.10, №2, С.376-386

SLIMANE A.

\title{
SPACES GENERATED BY THE CONE OF SUBLINEAR OPERATORS
}

\begin{abstract}
This paper deals with a study on classes of non linear operators. Let $S L(X, Y)$ be the set of all sublinear operators between two Riesz spaces $X$ and $Y$. It is a convex cone of the space $H(X, Y)$ of all positively homogeneous operators. In this paper we study some spaces generated by this cone, therefore we study several properties, which are well known in the theory of Riesz spaces, like order continuity, order boundedness etc. Finally, we try to generalise the concept of adjoint operator. First, by using the analytic form of Hahn-Banach theorem, we adapt the notion of adjoint operator to the category of positively homogeneous operators. Then we apply it to the class of operators generated by the sublinear operators.

Key words and phrases: Riesz space, Banach lattice, homogeneous operator, sublinear operator, order continuous operator.
\end{abstract}

Laboratory of functional analysis and geometry of spaces, University of M'sila, M'sila 28000, Algeria

E-mail: amr.slimane@gmail.com

\section{INTRODUCTION}

The theory of Riesz spaces plays an important role in several branches of mathematics, in particular in the geometry of Banach spaces and the theory of linear operators where the notion of Banach lattice play a central role. In this work we generalize some vector lattice properties to the category of sublinear operators i.e., positively homogenous and subadditive. The set obtained is not a Banach space but a positive convex cone. Hence, this paper deals with the extension of this set and their properties. The paper is organized as follows.

In Section 1 we recall some basic definitions and properties of Riesz spaces, we also recall the notion of sublinear operators between a vector space $X$ and a Riesz space $Y$.

In Section 2 we introduce the spaces spanned by different cones of sublinear operators. In other hand we present some principal notions concerning the theory of Riesz spaces like order continuity, order ideal, and we apply these notions on these spaces.

In Section 3 we introduce the adjoint of positively homogeneous operator. We first establish the following result.

Let $u$ be in $\mathcal{L}(X, Y)$. Then the bounded adjoint operator $u^{*}$ of $u$ can be extended to a bounded linear operator $\widetilde{u}^{*}$ belongs to $\mathcal{L}\left(H^{*}(Y), H^{*}(X)\right)$ such that $\widetilde{u}^{*}=u^{*}$ on $Y^{*}$ and $\left\|\widetilde{u}^{*}\right\|=$ $\left\|u^{*}\right\|=\|u\|$, where $H^{*}(Y)$ is the space of all bounded positively homogeneous functionals on $Y, Y^{*}$ is the topological dual space of $Y$ and $\mathcal{L}(X, Y)$ is the Banach space of all bounded linear operators from $X$ into $Y$. Finally we adapt the existence theorem of bounded adjoint linear operator to the category of positively homogeneous operators as follows.

$\mathrm{y} \Delta \mathrm{K} 517.983 .2$

2010 Mathematics Subject Classification: 46B42, 46B40, 47A05, 47B60, 47B65.

(C) Slimane A., 2018 
Let $X, Y$ be two Banach spaces and $T \in \mathcal{H}(X, Y)$. Then, $T_{h}^{*} \in \mathcal{L}\left(H^{*}(Y), H^{*}(X)\right)$ such that $\|T\|=\left\|T_{h}^{*}\right\|$, where $T_{h}^{*}$ denotes the adjoint of $T$ and $\mathcal{H}(X, Y)$ is the Banach space of all bounded positively homogeneous operators from $X$ into $Y$.

\section{PRELIMINARIES}

In this section, we introduce some terminology concerning Riesz spaces and Banach lattices. These spaces are well known. For more details, the interested reader can consult, for example, the references [2,4-6]. But for our convenience, we include some recalls. We also introduce the class of positively homogeneous operators.

Let $X$ be a real vector space. Then $X$ is called a Riesz space (or vector lattice) if it is an ordered vector space with the additional property that the supremum of every nonempty finite subset of $X$ exists in $X$. We denote the supremum of the set $\{x, y\}$ by $\sup \{x, y\}$ or $x \vee y$. Similarly, $\inf \{x, y\}$ or $x \wedge y$ denote the infimum of the set $\{x, y\}$.

Let $X$ be a Riesz space. The subset $X^{+}=\{x \in E: x \geq 0\}$ is called the positive cone of $X$ (which is salient, i.e. $\left.X^{+} \cap\left(-X^{+}\right)=\{0\}\right)$ and the elements of $X^{+}$are called the positive elements of $X$.

Let $X$ be a Riesz space, equipped with a norm. The norm in $X$ is called a Riesz norm if

$$
|x| \leq|y| \Longrightarrow\|x\| \leq\|y\|,
$$

where $|x|=\sup \{x,-x\}$. Denote $x^{+}=\sup \{x, 0\}, x^{-}=\sup \{-x, 0\}$. Then obviously we have $x=x^{+}-x^{-}$and $|x|=x^{+}+x^{-}$. Note that this implies that for any $x \in X$, the elements $x$ and $|x|$ have the same norm. A Riesz space $X$ equipped with a Riesz norm, is called a normed Riesz space. If the norm is complete, $X$ is called a Banach lattice. The convex cone $X^{+}$is norm closed. A complete Banach lattice is a Banach lattice such that every order bounded set in $X$ has a supremum.

By a Riesz subspace (or a vector sublattice) of a Riesz space $X$ we mean a linear subspace $E$ of $X$ so that $\sup \{x, y\}$ belongs to $E$ whenever $x, y \in E$. A vector subspace $E$ of a Riesz space $X$ is said to be an order ideal or simply ideal whenever $|x| \leq|y|$ and $y \in E$ imply $x \in E$.

A non-empty subset $D$ is said to be upwards directed (respectively downwards directed) if for all $x_{1}, x_{2} \in D$ there is $x_{3} \in D$ such that $x_{1} \vee x_{2} \leq x_{3}$ (respectively $x_{1} \wedge x_{2} \geq x_{3}$ ), if $\sup D=x$ exists and $D$ upwards directed (respectively inf $D=y$ exists and $D$ downwards directed) we shall write $D \uparrow x$ (respectively $D \downarrow y$ ).

Definition. Let $X$ be a vector space and $Y$ be a Riesz space. An operator $T: X \longrightarrow Y$ is

1 - positively homogeneous if for all $x$ in $X$ and $\lambda$ in $\mathbb{R}_{+}$we have

$$
T(\lambda x)=\lambda T(x),
$$

2- subadditive if for all $x, y$ in $X$ we have

$$
T(x+y) \leq T(x)+T(y) .
$$

The operator $T$ is sublinear if it is positively homogeneous and subadditive. The operator $T$ is said to be superlinear if $T$ is positively homogeneous and superadditive (i.e. $T(x+y) \geq$ $T(x)+T(y)$ for all $x, y$ in $X)$. We have for all $x$ in $X$

$$
-T(-x) \leq T(x)
$$


We denote by $H(X, Y)$ (respectively $S L(X, Y)$ ) the real vector space of all positively homogeneous (the set of all sublinear) operators from $X$ into $Y$, equipped with the natural order inducted by $Y$, i.e.

$$
T \leq S \quad \text { if } \quad T(x) \leq S(x), \quad \forall x \in X
$$

The set $S L(X, Y)$ is a pointed convex cone of $H(X, Y)$ which is not salient.

Let $T$ be in $S L(X, Y)$. We will denote by $\nabla T$ the subdifferential of $T$, which is the set of all linear operators $u: X \longrightarrow Y$ such that $u(x) \leq T(x)$ for all $x$ in $X$. We know (see, for example, [1]), that $\nabla T$ is not empty if $Y$ is a complete Banach lattice and $T(x)=\sup \{u(x): u \in \nabla T\}$, moreover, the supremum is attained. If $Y$ is simply a Banach lattice, then $\nabla T$ is empty in general (see [3]).

If $X$ is a Banach space and $Y$ is a Banach lattice, then we will denote by $\mathcal{S} \mathcal{L}(X, Y)$ the set of all bounded (= continuous) sublinear operators from $X$ into $Y$ and by $\mathcal{L}(X, Y)$ the Banach space of all bounded linear operators from $X$ into $Y$. Let $T$ be in $S L(X, Y)$. We have (see [1]), that $T$ is bounded if and only if $u$ is bounded for all $u$ in $\nabla T$. The set $\mathcal{S} \mathcal{L}(X, Y)$ (respectively the space $\mathcal{L}(X, Y)$ ) is a subset (respectively a subspace) of the space $\mathcal{H}(X, Y)$ of all homogeneous bounded operators from $X$ into $Y$. The space $\mathcal{H}(X, Y)$ is normed by the standard norm

$$
\|T\|=\sup _{\|x\| \leq 1}\|T(x)\| .
$$

\section{SPACES SPANNED BY SUbLINEAR OPERATORS}

Let $X$ be a vector space and $Y$ be a Riesz space. We denote by

$$
\triangle S L(X, Y)=S L(X, Y)-S L(X, Y)
$$

the subspace of $H(X, Y)$ spanned by $S L(X, Y)$, i.e.

$$
\triangle S L(X, Y)=\{T-S: T, S \in S L(X, Y)\} .
$$

We denote by $\triangle \mathcal{S} \mathcal{L}(X, Y)$ the subspace of all bounded operators in $\triangle S L(X, Y)$.

Proposition 1. Let $X$ be a vector space and $Y$ be a Riesz space. Then $H(X, Y)$ is a Riesz space. If in addition $X$ is a Banach space and $Y$ is a Banach lattice, then $\mathcal{H}(X, Y)$ is also a Banach lattice.

Proof. It is sufficent to endow the vector space $H(X, Y)$ with the partial order induced by $Y$. It is clear that $H(X, Y)$ is a Riesz space with respect to this order. Suppose now $X$ be a Banach space and $Y$ be a Banach lattice. Let $\left(T_{n}\right)_{n} \subset H(X, Y)$ be a Cauchy sequence, then $\lim _{n \rightarrow+\infty}\left\|T_{n+p}-T_{n}\right\|=0$ implies that $\lim _{n \rightarrow+\infty}\left\|T_{n+p}(x)-T_{n}(x)\right\|=0$ for all $x$ in $X$.

As $Y$ is a Banach space there is $T(x) \in Y$ such that $\lim _{n \rightarrow+\infty} T_{n}(x)=T(x)$. Since $T_{n}(\alpha x)=$ $\alpha T_{n}(x)$ for all $\alpha$ in $\mathbb{R}_{+}$and all $x$ in $X$ we have $T(\alpha x)=\lim _{n \rightarrow+\infty} T_{n}(\alpha x)=\lim _{n \rightarrow+\infty} \alpha T_{n}(x)=\alpha T(x)$ for all $\alpha$ in $\mathbb{R}_{+}$and all $x$ in $X$. Thus, $T$ is positively homogeneous. The operator $T$ is clearly bounded and hence $\mathcal{H}(X, Y)$ is a Banach space. Let now $T, S \in \mathcal{H}(X, Y)$ such that $|T| \leq|S|$ then $\|T(x)\| \leq\|S(x)\|$ for all $x$ in $X$, so $\|T\| \leq\|S\|$ and $\mathcal{H}(X, Y)$ is a Banach lattice. 
Proposition 2. Let $X$ be a vector space and $Y$ be a Riesz space. Then

(a) the space $\triangle S L(X, Y)$ is a Riesz subspace of $H(X, Y)$;

(b) if $X$ is a normed space and $Y$ be a normed Riesz space, then $\triangle \mathcal{S L}(X, Y)$ is a normed Riesz space.

Proof. (a) The space $\triangle S L(X, Y)$, which is included in $H(X, Y)$, is partially ordered by the natural order inducted by $Y$. Consider $T, S$ in $\triangle S L(X, Y)$. Then, there are $T_{1}, T_{2}, S_{1}, S_{2}$ in $S L(X, Y)$ such that

$$
T=T_{1}-T_{2}, S=S_{1}-S_{2} .
$$

For all $x$ in $X$ we define $T \vee S$ by

$$
(T \vee S)(x)=T(x) \vee S(x)
$$

Using for $x, y, z$ in $X$ the identity $x \vee y+z=(x+z) \vee(y+z)$, we obtain

$$
\begin{aligned}
(T \vee S)(x) & =\left(T_{1}-T_{2}\right)(x) \vee\left(S_{1}-S_{2}\right)(x) \\
& =\left(T_{1}+S_{2}\right)(x) \vee\left(S_{1}+T_{2}\right)(x)-\left(T_{2}+S_{2}\right)(x)=\widetilde{T}(x)-\widetilde{S}(x)
\end{aligned}
$$

with $\widetilde{T}, \widetilde{S} \in S L(X, Y)$, where

$$
\widetilde{T}=\left(T_{1}+S_{2}\right) \vee\left(S_{1}+T_{2}\right) \text { and } \widetilde{S}=T_{2}+S_{2} .
$$

(b) It is clear that $\triangle \mathcal{S} \mathcal{L}(X, Y)$ is a normed Riesz space with the norm induced by the standard norm of $\mathcal{H}(X, Y)$ on $\triangle \mathcal{S} \mathcal{L}(X, Y)$, i.e. by the norm $\|T\|_{\triangle \mathcal{S L}(X, Y)}=\sup _{\|x\| \leq 1}\|T(x)\|$.

Proposition 3. Let $X$ be a vector space and $Y$ be a Dedekind complete Riesz space. Then $H(X, Y)$ is also a Dedekind complete Riesz space.

Proof. Let $M \subset H(X, Y)$ be a nonempty subset, which is upper bounded. Then there is $S \in H(X, Y)$ such that for all $T \in M$ we have $T \leq S$, that is for all $T \in M$ and all $x \in X$ we have $T(x) \leq S(x)$. This implies that for all $x \in X$ the set $\{T(x): T \in M\}$ is upper bounded by $S(x) \in Y$. Since $Y$ is a Dedekind complete Riesz space, the supremum of $\{T(x): T \in M\}$ exists in $Y$. We can put now $R(x)=\sup \{T(x): T \in M\}$. It is clear that $R$ is a positively homogeneous operator.

Remark 1. For all $T=P-Q$ in $\triangle S L(X, Y)$ there is $\varphi_{T} \in S L(X, Y)$ and $\bar{\varphi}_{T}$ super linear (i.e. $-\bar{\varphi}_{T}$ sublinear) such that $\bar{\varphi}_{T} \leq T \leq \varphi_{T}$ and $\varphi_{T}(-x)=\varphi_{-T}(x)$ (respectively $\bar{\varphi}_{T}(-x)=$ $\left.\bar{\varphi}_{-T}(x)\right)$ for all $x$ in $X$. It suffices to define $\varphi_{T}, \bar{\varphi}_{T}$ by

$$
\varphi_{T}(x)=P(x)+Q(-x), \quad \bar{\varphi}_{T}(x)=-P(-x)-Q(x)
$$

and use the inequality (1).

Definition 1. Let $T \in \triangle S L(X, Y)$ be an operator between two Riesz spaces. The operator $T$ is said to be order bounded if $T$ carries order bounded subsets of $X$ to order bounded subsets of $Y$. 
Definition 2. Let $T \in \triangle S L(X, Y)$ be an order bounded operator. Then $T$ is said to be

(1) order continuous if for any downwards directed set $D$ in $E$ having infimum the null element (i.e. $D \downarrow 0$ ) we have $\inf (|T(x)|, x \in D)=0$ in $Y$;

(2) $\sigma$-order continuous if for all $x_{n} \downarrow 0$ in $X$ we have in $Y$

$$
\inf \left(\left|T\left(x_{n}\right)\right|, n \geq 0\right)=0 .
$$

We denote by

$$
\begin{aligned}
\triangle S L_{b}(X, Y) & =\{T \in \triangle S L(X, Y), T \text { order bounded }\}, \\
\triangle S L_{c o}(X, Y) & =\{T \in \triangle S L(X, Y), T \text { order continuous }\} .
\end{aligned}
$$

It should be clear that all these collections are real vector spaces under the usual pointwise algebraic operations.

Proposition 4. The set $\triangle S L_{b}(X, Y)$ is a Riesz subspace of $\triangle S L(X, Y)$.

Proof. Consider $T_{1}, T_{2}$ in $\triangle S L_{b}(X, Y),(\alpha, \beta)$ in $\mathbb{R}^{2}$ and $\alpha \leq x \leq \beta$. Then

$$
\left|\left(\alpha T_{1}+\beta T_{2}\right)(x)\right| \leq|\alpha|\left|T_{1}(x)\right|+|\beta|\left|T_{2}(x)\right| \leq|\alpha| c_{1}+|\beta| c_{2}=c .
$$

This implies that $\alpha T_{1}+\beta T_{2} \in \triangle S L_{b}(X, Y)$ and hence $T_{1} \vee T_{2} \in \triangle S L_{b}(X, Y)$ because $T_{1} \vee T_{2}=\frac{1}{2}\left(T_{1}+T_{2}+\left|T_{1}-T_{2}\right|\right)$. Consequently, $\triangle S L_{b}(X, Y)$ is a Riesz subspace of the Riesz space $\triangle S L(X, Y)$.

\section{THE ADJOINT OF POSITIVELY HOMOGENEOUS OPERATORS}

Definition 3. Let $X, Y$ be two Riesz spaces. Put

$$
\triangle_{r} S L(X, Y)=\left\{T_{1}-T_{2}: \quad T_{1}, T_{2} \in(S L(X, Y))^{+}\right\} \subset \triangle S L(X, Y) .
$$

A sublinear operator $T \in S L(X, Y)$ is said to be regular if $T \in \triangle_{r} S L(X, Y)$.

We denote by

$$
\begin{aligned}
S L_{i}(X, Y) & =\{T \in S L(X, Y): T \text { increasing }\} \\
\triangle S L_{i}(X, Y) & =\left\{T_{1}-T_{2}: \quad T_{1}, T_{2} \in S L_{i}(X, Y)\right\} \\
& =S L_{i}(X, Y)-S L_{i}(X, Y), \\
L_{i}(X, Y) & =\{T \in L(X, Y): \text { Tincreasing }\}, \\
\triangle L_{i}(X, Y) & =\left\{T_{1}-T_{2}: \quad T_{1}, T_{2} \in L_{i}(X, Y)\right\} \\
& =L_{i}(X, Y)-L_{i}(X, Y),
\end{aligned}
$$

and we put $X_{i}^{\prime}=\triangle L_{i}(X, \mathbb{R}), X_{i, s}^{\prime}=\triangle S L_{i}(X, \mathbb{R})$.

Proposition 5. The spaces $\triangle_{r} S L(X, Y), \triangle S L_{i}(X, Y)$ are Riesz subspaces of $\triangle S L(X, Y)$. 
Proof. The set $\triangle_{r} S L(X, Y)$ is a subspace of $\triangle S L(X, Y)$. Further, if $T_{1}, T_{2} \in \triangle_{r} S L(X, Y)$, then there is $P_{1}, Q_{1}, P_{2}, Q_{2} \in(S L(X, Y))^{+}$such that $T_{1}=P_{1}-Q_{1}$ and $T_{2}=P_{2}-Q_{2}$. We have $T_{1} \vee T_{2}=\left(P_{1}+Q_{2}\right) \vee\left(P_{2}+Q_{1}\right)-\left(Q_{1}+Q_{2}\right)$, which is in $\triangle_{r} S L(X, Y)$ because

$$
\left(P_{1}+Q_{2}\right) \vee\left(P_{2}+Q_{1}\right),\left(Q_{1}+Q_{2}\right) \in(S L(X, Y))^{+}
$$

The same for $\triangle S L_{i}(X, Y)$.

Proposition 6. The spaces $\triangle_{r} S L(X, Y), \triangle S L_{i}(X, Y)$ are Riesz subspaces of $\triangle S L(X, Y)$.

Proof. The set $\triangle_{r} S L(X, Y)$ is a subspace of $\triangle S L(X, Y)$. Further, if $T_{1}, T_{2} \in \triangle_{r} S L(X, Y)$, then there is $P_{1}, Q_{1}, P_{2}, Q_{2} \in(S L(X, Y))^{+}$such that $T_{1}=P_{1}-Q_{1}$ and $T_{2}=P_{2}-Q_{2}$. We have $T_{1} \vee T_{2}=\left(P_{1}+Q_{2}\right) \vee\left(P_{2}+Q_{1}\right)-\left(Q_{1}+Q_{2}\right)$, which is in $\triangle_{r} S L(X, Y)$ because

$$
\left(P_{1}+Q_{2}\right) \vee\left(P_{2}+Q_{1}\right),\left(Q_{1}+Q_{2}\right) \in(S L(X, Y))^{+}
$$

The same for $\triangle S L_{i}(X, Y)$.

Remark 2. 1) Any linear operator is a regular sublinear operator. Indeed, if $u \in L(X, Y)$, then $u=u^{+}-u^{-}$with $u^{+}(x)=0 \vee u(x), u^{-}(x)=0 \vee(-u(x))$, which are positive sublinear operators.

2) The existence of the regular sublinear operators (not linear) is assured by the fact that if $T \in S L(X, Y)$ such that $|T| \in S L(X, Y)$, then $T$ is regular

$$
T=T^{+}-T^{-}=2 T^{+}-|T| \quad\left(2 T^{+},|T| \in(S L(X, Y))^{+}\right) .
$$

As example, consider $\alpha, \beta \in \mathbb{R}^{+}$such that $\alpha>\beta$ and $T: \mathbb{R} \longrightarrow \mathbb{R}$ defined by

$$
T(x)= \begin{cases}\alpha x, & \text { if } x \geq 0 \\ \beta x, & \text { if } x<0\end{cases}
$$

Then $T$ is sublinear $(T(x)=(\alpha x) \vee(\beta x))$ and $|T|$ also because

$$
|T|(x)=|T(x)|=(\alpha x) \vee(-\beta x) .
$$

Lemma 1 ([6, Lemma 21.3]). Let $E$ be an ordered vector space, and let $A, B$ be two subsets of $E$ such that $\inf A=x_{0}$, inf $B=y_{0}$. Then

$$
x_{0}+y_{0}=\inf (A+B)=\inf \{a+b \text { such that } a \in A, b \in B\} \text {. }
$$

Proposition 7. Let X, Y be two Riesz spaces. Put

$$
\begin{aligned}
S L_{o}(X, Y) & =\left\{T \in S L_{i}(X, Y) \text { such that } T \text { order continuous }\right\}, \\
\triangle S L_{o}(X, Y) & =S L_{o}(X, Y)-S L_{o}(X, Y) .
\end{aligned}
$$

Then

(a) the set $S L_{o}(X, Y)$ is a convex cone;

(b) the space $\triangle S L_{o}(X, Y) \subset \triangle S L_{c o}(X, Y)$ is an order ideal. 
Proof. (a) Let $D \downarrow 0$, and $p, q \in S L_{o}(X, Y)$, then $(p+q)(D)$ is upwards directed such that $(p+q)(D) \downarrow 0$. Indeed, if $x_{1}, x_{2} \in D$, then there is $x_{3} \in D$ such that $x_{3} \leq x_{1}$ and $x_{3} \leq x_{2}$. This implies that $(p+q)\left(x_{3}\right) \in(p+q)(D)$. Thus

$$
(p+q)\left(x_{3}\right) \leq(p+q)\left(x_{1}\right) \text { and }(p+q)\left(x_{3}\right) \leq(p+q)\left(x_{2}\right) .
$$

Let $h$ be the infimum of $(p+q)(D)$, then for all $x_{1}, x_{2} \in D$ there is $x_{3} \in D$ such that

$$
h \leq(p+q)\left(x_{3}\right) \leq p\left(x_{1}\right)+q\left(x_{2}\right) \text { for all } x_{1}, x_{2} \in D .
$$

We have

$$
\begin{aligned}
h & \leq \inf \left\{p\left(x_{1}\right)+q\left(x_{2}\right), x_{1}, x_{2} \in D\right\} \\
& \leq \inf \left\{p\left(x_{1}\right), x_{1} \in D\right\}+\inf \left\{q\left(x_{2}\right), x_{2} \in D\right\} \\
& \leq \inf \left\{\left|p\left(x_{1}\right)\right|, x_{1} \in D\right\}+\inf \left\{\left|q\left(x_{2}\right)\right|, x_{2} \in D\right\} \leq 0 .
\end{aligned}
$$

Consequently,

$$
\begin{aligned}
\inf \{|(p+q)(x)|, x \in D\} & \leq \inf \{|p(x)|+|q(x)|, x \in D\} \\
& \leq \inf \{p(x)+q(x), x \in D\} \leq 0 .
\end{aligned}
$$

It is clear that $\lambda p \in S L_{o}(X, Y)$ for all $\lambda \in \mathbb{R}^{+}$and all $p \in S L_{o}(X, Y)$. Furthermore

$$
\begin{aligned}
\inf \{|(p \vee q)(x)|, x \in D\} & =\inf \{(p \vee q)(x), x \in D\} \\
& \leq \inf \{(p+q)(x), x \in D\} \leq 0 .
\end{aligned}
$$

(b) Let $T \in \triangle S L_{o}(X, Y)$. Then $T=p-q$ with $p, q \in S L_{o}(X, Y)$. Let $D \downarrow 0$. We have

$$
|p-q|(x) \leq|p(x)|+|q(x)| \leq p(x)+q(x) \text { for all } x \in D \text {. }
$$

So,

$$
\inf \{|(p-q)(x)|, x \in D\} \leq \inf \{(p+q)(x), x \in D\} \leq 0 .
$$

Consequently, $T \in \triangle S L_{c o}(X, Y)$.

Let now $D \downarrow 0$. Assume that $|T| \leq|S|, S \in \triangle S L_{o}(X, Y)$, then

$$
\inf \{|T|(x), x \in D\} \leq \inf \{|S|(x), x \in D\} \leq 0 .
$$

This ends the proof.

In the sequel, we extend the notion of adjoint operator on some spaces defined above. Let $X$ be a Banach space and $Y$ be a Banach lattice. Put

$$
\begin{aligned}
X^{\prime} & =L(X, \mathbb{R}), \\
X^{*} & =\mathcal{L}(X, \mathbb{R}), \\
X_{\Delta}^{\prime} & =\triangle S L(X, \mathbb{R}), \\
X_{\Delta}^{*} & =\triangle \mathcal{S} \mathcal{L}(X, \mathbb{R}), \\
H^{\prime}(X) & =H(X, \mathbb{R}), \\
H^{*}(X) & =\mathcal{H}(X, \mathbb{R}) .
\end{aligned}
$$

We have $X^{\prime} \subset X_{\Delta}^{\prime} \subset H^{\prime}(X)$ and $X^{*} \subset X_{\Delta}^{*} \subset H^{*}(X)$. 
Theorem 1. Let $X, Y$ be two Riesz spaces and $u$ be in $L(X, Y)$. Then there exists an $\widetilde{u}^{\prime}$ in $L\left(H^{\prime}(Y), H^{\prime}(X)\right)$ such that $\widetilde{u}^{\prime}=u^{\prime}$ on $Y^{\prime}$ and $\widetilde{u}^{\prime}(\varphi) \leq|\varphi \circ u|$ for all $\varphi \in H^{\prime}(Y)$, where $u^{\prime}$ is the adjoint operator of $u$.

Proof. Let $u$ be in $L(X, Y)$, the adjoint operator of $u$ is defined by

$$
u^{\prime}: Y^{\prime} \longrightarrow X^{\prime} \subset H^{\prime}(X)
$$

such that

$$
u^{\prime}(\varphi)=\varphi \circ u \text { for all } \varphi \in Y^{\prime} .
$$

Let now $P \in S L\left(H^{\prime}(Y), H^{\prime}(X)\right)$ be defined by

$$
P(\varphi)=|\varphi \circ u| \text {. }
$$

We have

$$
u^{\prime}(\varphi)=\varphi \circ u \leq|\varphi \circ u|=P(\varphi) \text { for all } \varphi \in Y^{\prime} .
$$

By the Hahn-Banach theorem (the analytic form), there is $\widetilde{u}^{\prime} \in L\left(H^{\prime}(Y), H^{\prime}(X)\right)$ such that $\widetilde{u}^{\prime}=u^{\prime}$ on $Y^{\prime}$ and

$$
\widetilde{u}^{\prime}(\varphi) \leq P(\varphi) \leq|\varphi \circ u|
$$

for all $\varphi \in H^{\prime}(Y)$ and this completes the proof.

Theorem 2. Let $X, Y$ be two Banach spaces and $u$ be in $\mathcal{L}(X, Y)$. Then there exists an $\widetilde{u}^{\prime}$ in $\mathcal{L}\left(H^{*}(Y), H^{*}(X)\right)$ such that $\widetilde{u}^{\prime}=u^{*}$ on $Y^{*}$ and $\left\|\widetilde{u}^{\prime}\right\|=\left\|u^{*}\right\|=\|u\|$. In this case $\widetilde{u}^{\prime}$ is denoted by $\widetilde{u}^{*}$.

Proof. Let $u$ be in $\mathcal{L}(X, Y)$. By Theorem 1 there is $\widetilde{u}^{\prime}$ in $L\left(H^{\prime}(Y), H^{\prime}(X)\right)$ such that $\widetilde{u}^{\prime}=u^{*}$ on $Y^{\prime}$ and $\widetilde{u}^{\prime}(\varphi) \leq|\varphi \circ u|$ for all $\varphi \in H^{\prime}(Y)$. On the other hand, because $\widetilde{u}^{\prime}(\varphi) \leq|\varphi \circ u|$ we obtain $\left|\widetilde{u}^{\prime}(\varphi)\right| \leq|\varphi \circ u|$ and hence for all $\varphi \in H^{*}(Y)$

$$
\left\|\widetilde{u}^{\prime}(\varphi)\right\| \leq\|\varphi \circ u\| \leq\|u\|\|\varphi\| .
$$

So, $\widetilde{u}^{\prime} \in \mathcal{L}\left(H^{*}(Y), H^{*}(X)\right)$. It remains to show that $\left\|\widetilde{u}^{\prime}\right\|=\|u\|$. Since $\left\|\widetilde{u}^{\prime}(\varphi)\right\| \leq\|u\|\|\varphi\|$, we conclude that $\left\|\widetilde{u}^{\prime}\right\| \leq\|u\|$. For the converse inequality, we know that $\left\|u^{*}\right\|=\|u\|$, hence

$$
\begin{aligned}
\|u\|=\left\|u^{*}\right\| & =\sup _{\varphi \in B_{\Upsilon^{*}}}\left\|u^{*}(\varphi)\right\| \\
& \left.=\sup _{\varphi \in B_{\Upsilon^{*}}}\left\|\widetilde{u}^{\prime}(\varphi)\right\| \text { (because } \widetilde{u}^{\prime} / Y^{*}=u^{*}\right) \\
& \left.\leq \sup _{\varphi \in B_{H^{*}(Y)}}\left\|\widetilde{u}^{\prime}(\varphi)\right\| \text { (because } B_{Y^{*}} \subset B_{H^{*}(Y)}\right) \\
& =\left\|\widetilde{u}^{\prime}\right\|
\end{aligned}
$$

and then the theorem is proved.

Now, we extend the notion of adjoint operator to positively homogeneous operators.

Definition 4. Let $X, Y$ be two Riesz spaces and $T \in H(X, Y)$. We define the adjoint of $T$ by

$$
\begin{aligned}
T_{h}^{\prime}: H^{\prime}(Y) & \longrightarrow H^{\prime}(X) \\
\varphi & \longmapsto T_{h}^{\prime}(\varphi)=\varphi \circ T
\end{aligned}
$$

such that $T_{h}^{\prime}(\varphi)(x)=\varphi \circ T(x)$. 
Proposition 8. Let $X, Y$ be two Banach spaces and $T \in \mathcal{H}(X, Y)$. Then $T_{h}^{\prime} \in \mathcal{L}\left(H^{*}(Y), H^{*}(X)\right)$ such that $\|T\|=\left\|T_{h}^{\prime}\right\|$. In this case $T_{h}^{\prime}$ is denoted by $T_{h}^{*}$.

Proof. Consider $T$ in $\mathcal{H}(X, Y)$. We have for all $\varphi \in H^{*}(Y)$

$$
\left\|T_{h}^{\prime}(\varphi)\right\|=\|\varphi \circ T\| \leq\|\varphi\|\|T\| .
$$

So, $T_{h}^{\prime} \in \mathcal{L}\left(H^{*}(Y), H^{*}(X)\right)$. To show that $\|T\|=\left\|T_{h}^{\prime}\right\|$, we first consider the mapping $i: x \in X \longmapsto i(x) \in H^{* *}(X)$ such that

$$
\begin{aligned}
i(x): H^{*}(X) & \longrightarrow \mathbb{R}, \\
\varphi & \longmapsto(i(x), \varphi)=\langle\varphi, x\rangle .
\end{aligned}
$$

Then $i$ is such that $\|i(x)\|=\|x\|$ for all $x \in X$. Indeed,

$$
\begin{aligned}
\|i(x)\| & =\sup _{\varphi \in B_{H^{*}(X)}}\|(i(x), \varphi)\| \\
& =\sup _{\varphi \in B_{H^{*}(X)}}\|\langle\varphi, x\rangle\| \\
& \leq\|x\| .
\end{aligned}
$$

Conversely

$$
\begin{aligned}
\|x\| & \left.=\sup _{\xi \in B_{X^{*}}}\|\langle\xi, x\rangle\| \leq \sup _{\varphi \in B_{H^{*}(X)}}\|\langle\varphi, x\rangle\| \text { (because } B_{X^{*}} \subset B_{H^{*}(X)}\right) \\
& \leq \sup _{\varphi \in B_{H^{*}(X)}}\|(i(x), \varphi)\| \leq\|i(x)\| .
\end{aligned}
$$

Finally, we have

$$
\begin{aligned}
\left\|T_{h}^{\prime}\right\|=\sup _{\varphi \in B_{H^{*}(Y)}\left\|T_{h}^{\prime}(\varphi)\right\|}=\sup _{\varphi \in B_{H^{*}(Y)}}\|\varphi \circ T\| \\
=\sup _{\varphi \in B_{H^{*}(Y)}}\left(\sup _{x \in B_{X}}\|\langle\varphi \circ T, x\rangle\|\right) \\
=\sup _{\varphi \in B_{H^{*}(Y)}}\left(\sup _{x \in B_{X}}\|\langle\varphi, T(x)\rangle\|\right) \\
=\sup _{x \in B_{X}}\left(\sup _{\varphi \in B_{H^{*}(Y)}}\|\langle\varphi, T(x)\rangle\|\right) \\
=\sup _{x \in B_{X}}\left(\sup _{\varphi \in B_{H^{*}}(Y)}\|(i(T(x)), \varphi)\|\right) \\
=\sup _{x \in B_{X}}\|i(T(x))\| \\
=\sup _{x \in B_{X}}\|T(x)\|=\|T\| .
\end{aligned}
$$

This completes the proof.

Definition 5. Let $X, Y$ be two Riesz spaces. Consider $T \in \triangle S L(X, Y)$ with $T=P-Q$. We define a linear operator on $Y_{i, s}^{\prime}$ denoted $T_{i}^{\prime}$ by

$$
\begin{aligned}
& T_{i}^{\prime}: Y_{i, s}^{\prime} \longrightarrow X_{\Delta \prime}^{\prime} \\
& T_{1}-T_{2} \longmapsto T_{i}^{\prime}\left(T_{1}-T_{2}\right)=T_{1} \circ P+T_{2} \circ Q-\left(T_{1} \circ Q+T_{2} \circ P\right) .
\end{aligned}
$$


Note that this operator is well defined. Indeed, if $S \in Y_{i, S}^{\prime}$ such that $S=S_{1}-S_{2}=S_{3}-S_{4}$, then

$$
\begin{aligned}
T_{i}^{\prime}\left(S_{1}-S_{2}\right) & =S_{1} \circ P+S_{2} \circ Q-\left(S_{1} \circ Q+S_{2} \circ P\right) \\
& =\left(S_{1}-S_{2}\right) \circ P-\left(S_{1}-S_{2}\right) \circ Q \\
& =\left(S_{3}-S_{4}\right) \circ P-\left(S_{3}-S_{4}\right) \circ Q=T_{i}^{\prime}\left(S_{3}-S_{4}\right) .
\end{aligned}
$$

Proposition 9. Let $X, Y$ be two Riesz spaces, then there is $\widetilde{T}_{i}^{\prime}$ in $L\left(H^{\prime}(Y), H^{\prime}(X)\right)$ such that $\widetilde{T}_{i}^{\prime}=T_{i}^{\prime}$ on $Y_{i, s}^{\prime}$.

Proof. We define a sublinear operator $S: H^{\prime}(Y) \longrightarrow H^{\prime}(X)$ by

$$
S(\varphi)=|\varphi \circ P|+|\varphi \circ Q| .
$$

For all $\varphi=\varphi_{1}-\varphi_{2} \in Y_{i, S}^{\prime}$ we have

$$
\begin{aligned}
T_{i}^{\prime}(\varphi)=T_{i}^{\prime}\left(\varphi_{1}-\varphi_{2}\right) & =\varphi_{1} \circ P+\varphi_{2} \circ Q-\left(\varphi_{1} \circ Q+\varphi_{2} \circ P\right)=\left(\varphi_{1}-\varphi_{2}\right) \circ P-\left(\varphi_{1}-\varphi_{2}\right) \circ Q \\
& \leq\left|\left(\varphi_{1}-\varphi_{2}\right) \circ P\right|+\left|\left(\varphi_{1}-\varphi_{2}\right) \circ Q\right|=S(\varphi) .
\end{aligned}
$$

The Hahn-Banach theorem implies that $T_{i}^{\prime}$ can be extended to a linear operator $\widetilde{T}_{i}^{\prime} \in L\left(H^{\prime}(Y), H^{\prime}(X)\right)$ such that $\widetilde{T}_{i}^{\prime}(\varphi) \leq S(\varphi)$ for all $\varphi \in H^{\prime}(Y)$.

Remark 3. If $T \in L(X, Y)$, then we have $\widetilde{T}^{\prime}=T_{h}^{*}$ on $Y^{\prime}$, where $T_{h}^{\prime}$ denote the operator defined in Definition 4. If $T \in \triangle S L(X, Y)$, then we have $\widetilde{T}_{i}^{\prime}=T_{h}^{\prime}$ on $Y_{i}^{\prime}$.

Proposition 10. Let $X, Y$ be two Riesz spaces and $T$ be in $(S L(X, Y))^{+}$. Then the following properties are satisfied.

(1) We have $|T|_{i}^{\prime} \leq\left|T_{i}^{\prime}\right|$.

(2) The restriction of $T_{i}^{\prime}$ to $S L_{i}(Y, \mathbb{R})$ verifies $\left|T_{i}^{\prime}\right|=|T|_{i}^{\prime}$.

Proof. (1) Let $T \in(S L(X, Y))^{+}$and $\varphi \in Y_{i, S^{\prime}}^{\prime}$ then there is $\varphi_{1}, \varphi_{2} \in S L_{i}(X, Y)$ such that $\varphi=\varphi_{1}-\varphi_{2}$ and

$$
\begin{aligned}
\left|T_{i}^{\prime}\right|(\varphi) & =\left|T^{\prime}(\varphi)\right|=\left|\varphi_{1} \circ T-\varphi_{2} \circ T\right| \geq \varphi_{1} \circ T-\varphi_{2} \circ T \\
& \geq \varphi_{1} \circ|T|-\varphi_{2} \circ|T| \geq|T|_{i}^{\prime}(\varphi) .
\end{aligned}
$$

(2) Let $T \in(S L(X, Y))^{+}$and $\varphi \in S L_{i}(Y, \mathbb{R})$ we have

$$
\begin{aligned}
\left|T_{i}^{\prime}\right|(\varphi) & =\left|T^{\prime}(\varphi)\right|=|\varphi(T)|=\varphi(T)(\text { because } \varphi \uparrow \text { and } T \geq 0) \\
& =\varphi(|T|)=|T|_{i}^{\prime}(\varphi)
\end{aligned}
$$

and this completes the proof. 


\section{REFERENCES}

[1] Achour D., Mezrag L. Little Grothendieck's theorem for sublinear operators. J. Math. Anal. Appl. 2004,296 (2), 541-552. doi: 10.1016/j.jmaa.2004.04.018

[2] Aliprantis C.D., Burkinshaw O. Positive operators. Springer, Netherlands, 1985.

[3] Linke Y.E. Sublinear operators without subdifferentials. Sibir. Math. Zh. 1991, 32 (3), $219-221$. doi: $10.1007 /$ BF00970495

[4] Meyer-Nieberg P. Banach lattices. Springer-Verlag, Berlin, Heidelberg, New-York, 1991.

[5] Schaefer H.H. Topological vector spaces. Springer-Verlag, New-York, Heidelberg, Berlin, 1970.

[6] Zaanen A.C. Introduction to operator theory in Riesz spaces. Springer-Verlag, Berlin, Heidelberg, New-York, 1991.

Received 19.12.2017

Слімане А. Простори, породжені конусом сублінійних операторів // Карпатські матем. публ. 2018. — T.10, №2. - C. 376-386.

У цій статті досліджуються деякі класи нелінійних операторів. Нехай $S L(X, Y)$ - множина всіх сублінійних операторів між двома просторами Ріса $X$ та $Y$. Це $є$ опуклий конус в просторі $H(X, Y)$ всіх позитивно однорідних операторів. У цій статті досліджено деякі простори, породжені цим конусом, зокрема ми досліджуємо деякі властивості, які добре відомі в теорії просторів Ріса, такі як порядкова неперервність, порядкова обмеженість та ін. Насамкінець, ми пробуємо узагальнити концепцію спряженого оператора. Спочатку, використовуючи аналітичну форму теореми Гана-Банаха, ми пристосовуємо поняття спряженого оператора до категорії позитивно однорідних операторів, а потім застосовуємо його до класу операторів, породжених сублінійними операторами.

Ключові слова і фрази: простір Ріса, банахова гратка, однорідний оператор, сублінійний оператор, порядково неперервний оператор. 